\title{
Advances in the Relationship between Tau Protein and Morphine De- pendence in Cognitive Dysfunction
}

\author{
Qing Ji, Xin Li*
}

The First Affiliated Hospital of Jiamusi University, Jiamusi 154003, China. E-mail: Arashijq@ 163.com

\begin{abstract}
Morphine is an opioid drug. Long-term use can cause morphine dependence or addiction, and there are cognitive dysfunction such as abnormal mental behavior, decline in learning and memory, and decline in executive ability. The occurrence of this disease is related to many factors, such as oxidative stress, hippocampal neuronal injury, mitochondrial function injury, etc. Tau protein is a microtubule-associated protein involved in nervous system development. Studies have found that hyperphosphorylation of tau proteins can cause apoptosis of hippocampal neurons ${ }^{[1]}$, and tau proteins can cause oxidative stress ${ }^{[2]}$. Therefore, tau proteins play an important role in the pathogenesis of cognitive disorders. The relationship between morphine dependence and cognitive dysfunction is now reviewed.
\end{abstract}

Keywords: Tau Protein; Morphine Dependence; Cognitive Impairment; Correlation

Morphine has a strong analgesic effect and is often used in anaesthesia and severe pain caused by terminal tumors. Long-term use of morphine can produce a strong dependence, leading to cognitive dysfunction, mental disorders, behavioral disorders and so on, and eventually cause psychological and physical damage to patients, and can lead to family rupture, which poses a threat to social stability. Cognitive impairment in patients with cognitive dysfunction caused by morphine dependence is mainly manifested in cognitive flexibility and working memory; cognitive flexibility is mainly reflected in selective attention, executive function, and working memory load ability. Reduced working memory load can cause a decline in decision-making ability ${ }^{[3-4]}$. The mechanism of cognitive dysfunction caused by morphine dependence is generally considered to be associated with mechanisms such as oxidative stress, hippocampal neuronal damage ${ }^{[3-4]}$, apoptosis, abnormal secretion of monoamine neuro- transmitters, altered synaptic plasticity, and mitochondrial dysfunction. However, the specific pathogenesis remains unclear. Tau protein is a tubulin involved in the regulation of tubulin stability ${ }^{[3]}$.

Accumulation of neuronal fiber tangles caused by hyperphosphorylation of tau proteins is closely related to the characteristic manifestations of cognitive dysfunction in Alzheimer's disease. Morphine is an opioid, long-term use can cause morphine dependence or addiction, and there are mental and behavioral disorders, learning and memory decline, executive ability decline and other cognitive dysfunction ${ }^{[4]}$. Recent studies have confirmed that acute and chronic morphine treatment can cause abnormal hyperphosphorylation of tau proteins and nerve microfilaments in the cerebral cortex ${ }^{[5-6]}$. As a result tau proteins may be associated with cognitive impairment following morphine dependence. This article reviews the

\footnotetext{
Copyright (C) 2020 Qing Ji et al.

doi: 10.18686/aem.v9i4.178

This is an open-access article distributed under the terms of the Creative Commons Attribution Non-Commercial License

(http://creativecommons.org/licenses/by-nc/4.0/), which permits unrestricted non-commercial use, distribution, and reproduction in any medium, provided the original work is properly cited
} 
overview of tau proteins and their phosphorylation regulation, tau proteins and other cognitive disorders related diseases, tau proteins and morphine dependence, tau proteins may be involved in regulating the pathogenesis of cognitive dysfunction caused by morphine dependence, in order to provide new ideas for the treatment and prevention of cognitive dysfunction caused by morphine dependence.

\section{Overview and phosphorylation regulation of tau proteins}

\subsection{Overview of tau proteins}

Tau protein is a microtubule related protein, which can be involved in regulating the stability of tubulin. Human tau genes on chromosome 17(17 q21-q22). And there are six tau isomers in the brain, is caused by or without exon 2, 3/10 messenger mRNA composed of alternative splicing. Exon 10 contains a bule binding region, exon 10 insertion provides four repeat ( $4 \mathrm{R}$ ) tau isomers, and 3 repeat $(3 \mathrm{R})$ tau isomers do not require the involvement of exon $10^{[5]}$. The adult brain mainly expresses $3 \mathrm{R}$ and $4 \mathrm{R}$ tau, under physiological conditions, they are mainly distributed in axons of neurons ${ }^{[6]}$. Neuronal fiber tangles composed of hyperphosphorylation of tau proteins are closely related to the main pathogenic causes of Alzheimer's disease ${ }^{[7]}$. Diseases occur around the terminal binding sites of tubulin $\mathrm{C}$, which leads to the decrease of microtubule binding ability and the weakening of microtubule binding ability, indicating that microtubule regulation is impaired ${ }^{[8-9]}$. Tau with localized misdistribution can also lead to impaired microtubule regulatory function. Mislocalized tau can be hyperphosphorylated while forming fibrous seeds and deposits. Furthermore, the stability of hyperphosphorylated proteins and normal tau proteins increased. Abnormal action of filamentous actin with stable tau proteins can lead to prominent damage, mitochondrial integrity defects, and actin instability. As a result, pathological changes in tau proteins can damage cytoskeleton system, cell transport system, mitochondrial integrity and signal transduction system. As a result, tau proteins accumulate in a group of brain cells and transfer to other regions can lead to disease and neurodegeneration.

\subsection{Phosphorylation regulation of tau pro- teins}

Tau protein is a phosphoprotein whose phosphorylation state determines its function. The binding of phosphate group depends not only on the conformation of tau protein, but also on the balance between tau protein kinase and protein phosphatase activity. Normal tau proteins usually bind 2-3 phosphatases, and when the protein kinase and protein phosphatase are out of balance, tau protein-bound phosphatases become 5-9. Current research finds ${ }^{[8]}$, there are 21 tau protein phosphorylation sites in Alzheimer's disease (AD) patients, protein kinase (PDPK) and non-proline guided protein kinase (non-PDPK) phosphorylation can be guided by proline. PDPK phosphorylation of serine and threonine containing Ser/Thr-pro sequences, including cell cycle dependent protein-5(CDK-5), glycogen synthesis kinase-3(GSK-3), mitogen-activated protein kinase (MAPK), the non-PDPK is mainly about Ser/Thr-X contained the serine of the sequence is phosphorylated with threonine, including calmodulin dependent protein kinase II (CaMK II), microtubule binding regulatory kinase (MARK) and cAMP dependent protein kinase (PKA), etc. Moreover ${ }^{[9]}$, protein phosphatase plays an important role PP2A regulating tau protein phosphorylation. The phosphorylation of Thr1620/1623 and Ser136 at the two sites of MAP-2 is mainly regulated by CaMK II and GSK-3 $\beta$, and PP2A plays an important role in dephosphorylation. Studies ${ }^{[10]}$ have confirmed that the up-regulation of tau and MAP-2 phosphorylation levels in acute morphine mainly by inhibiting PP2A activity, while the activation of tau and MAP-2 phosphorylation in chronic morphine by enhancing GSK-3 $\beta$ and PKA activity sex. Patrick found that P25/CDK5 complexes can not only induce hyperphosphorylation of tau proteins, but also reduce the ability of tau proteins to bind to microtubules. Wang Xinbo ${ }^{[1]}$ and others confirmed that Anshen Dingzhi can inhibit tau protein phosphorylation by activating BDNF/TrKB signal transduction pathway, thus improving the learning and memory ability of $\mathrm{AD}$ rats.

\section{Tau proteins and other cognitive disorders}

The biological activity of tau protein is the basis of maintaining its function. Moderate phosphorylation can maintain the biological activity of tau protein, but hy- 
perphosphorylation can cause tau protein to accumulate and form neuronal fiber tangles (NFT), thus losing its biological activity. The study found that tau protein phosphorylation changes in Alzheimer's disease, Parkinson's disease, stroke with vascular cognitive dysfunction, post-operative cognitive impairment and other cognitive impairment-related diseases, while tau protein phosphorylation can cause a decline in microtubule assembly ability. It can also further damage microtubules through tubulin-related protein MAP1 and MAP2, affecting the synthesis, transport, release of neurotransmitters resulting in abnormal intercellular signal transduction. Hence, hyperphosphorylation of tau proteins is thought to be involved in regulating the pathogenesis of multiple cognitive impairment-related diseases.

\subsection{Tau proteins and Alzheimer's disease}

The characteristic lesion of Alzheimer's disease $(\mathrm{AD})^{[12]}$ neurofibrillary tangles (NFTs) is an important cause of neuronal lesions, which can be used as a marker of brain aging and is positively related to the degree of cognitive impairment in AD. Tau protein can produce a large number of abnormal post-translational modifications and aggregate to form paired helical filaments, NFTs is formed by further deposition of paired helical filaments. Phosphorylation and interception are the main forms of post-translational modification of tau proteins, and are closely related to the occurrence and development of $\mathrm{AD}$. The degree of tau protein phosphorylation in the brain of $\mathrm{AD}$ patients is 3-4 times higher than that of ordinary people ${ }^{[13]}$. Tau protein phosphorylation is mainly regulated by phosphatase and phosphokinase to maintain the equilibrium state. When the equilibrium state is broken, it can cause abnormal hyperphosphorylation of tau protein, and hyperphosphorylation improves the aggregation ability of tau protein significantly. Loss of original function, accumulation in the form of spiral filaments NFTs, resulting in neuronal degeneration damage.

\subsection{Tau protein and Parkinson's disease}

Parkinson's disease (PD) is a common dyskinesia disease, the main pathological features of which are progressive neuronal degeneration in striatum and corpus callosum, and the widespread existence $\alpha$ - synaptic nucleoprotein aggregates-Louis corpuscles in cytoplasm.
Where cognitive dysfunction is PD common nonmotor symptom, including Parkinson's disease dementia (PDD) and Parkinson's disease mild cognitive impairment (PD-MCI). The results showed ${ }^{[14]}$ that the genotypes H1/H2 microtubule related tau protein (MAPT) genes and apolipoprotein Ec alleles were correlated with the dementia rate of Parkinson's disease. Cerebrospinal fluid $\beta$ - amyloid42 decreased, phosphorylated tau protein and total tau protein levels ${ }^{[15]}$ increased in PDD patients. Moreover, it was found that the tau level of serum phosphorylation in PD patients was significantly higher than that in the control group, and the level of serum phosphorylation tau in PD-MCI group was significantly lower than that in PDD group. The higher the serum tau protein phosphorylation level, the worse the prognosis.

\subsection{Tau proteins and post-stroke cognitive impairment}

Cognitive dysfunction after stroke belongs to vascular cognitive dysfunction (VCI), which refers to the impairment of one or more cognitive fields such as executive ability, language comprehension calculation, brain memory, structural ability and expression application. The study suggests that ${ }^{[16]} \beta$ - abnormal proteins produced during stroke such as amyloid 1-42(A $\beta-42)$ and tau protein may be associated with vascular cognitive dysfunction after stroke. Other studies have suggested that ${ }^{[17]}$ tau proteins are related to the formation of microtubules in neuronal cells and are specific proteins in the nervous system. After stroke, tau are released from nerve cells into cerebrospinal fluid and leak to peripheral blood through damaged blood-brain barrier. Its level may be related to the degree of brain injury. The results showed that ${ }^{[18]}$ the $A \beta-42$ of tau protein in the experimental group was lower than that in the control group, and the difference was statistically significant. The tau protein level was negatively correlated with the MoCA and MMSE score, suggesting that the $A \beta-42$ and tau protein level was associated with vascular cognitive impairment after stroke.

\section{Tau protein and morphine de- pendence}

Morphine is an opioid that can lead to cognitive decline such as learning and memory, but the specific reasons are unknown. Both acute and chronic morphine 
treatment can increase the phosphorylation of tau protein and nerve microfilament in rat cerebral cortex. Recent studies have confirmed that acute morphine treatment can increase the expression of cycle-dependent protein kinase-5 (CDK5) in cerebral cortex cells, and CDK5 is one of the important kinases that catalyze the phosphorylation of tau protein and neurofilament protein. Increased CDK5 activity can cause abnormal hyperphosphorylation of tau protein and neurofilament protein. But other studies have different views. Narita ${ }^{[19]}$ contet and other studies found that there was no significant change in the expression level of cerebral cortex CDK5 chronic morphine treatment, but the expression level of p-CDK5 (Ser159) increased and the activity of CDK5 increased. Suggesting that abnormal hyperphosphorylation of tau proteins and neurofilament proteins may be associated with increased p-CDK5 (ser159) expression levels and upregulation of CDK5 activity. Studies have also confirmed that ${ }^{[20]}$ high dose exogenous CCK-8 can significantly reduce the damage of spatial reference memory in morphine dependent mice, and CCK- 8 intervention can increase the PKA and GSK-3 $\beta$ activity caused by increasing the PP2A activity caused by acute morphine action and decreasing the chronic morphine action. It inhibited the increase of tau and MAP-2 phosphorylation induced by morphine.

\section{Mechanisms of possible involve- ment of tau proteins in mor- phine-dependent cognitive impair- ment}

Tau protein affects cognitive dysfunction caused by morphine dependence. A number of studies have found that tau proteins can not only affect apoptosis, but also affect nervous system and cognitive function through other ways. At present, the research includes the following aspects.

\subsection{Tau proteins affect apoptosis}

Apoptosis is an important regulatory mechanism to maintain the relative stability of physiological state. Its abnormal increase or decrease may lead to the change of function or structure of tissues and organs. The effects ${ }^{[21]}$ of stilbene glycoside (TSG) on the phosphorylation of tau protein in NG108-15 cells induced by okadaic acid
(OA) were studied. It was found that OA could lead to abnormal phosphorylation of tau protein in nerve cells and increase the aggregation of tau protein. Finally, apoptosis was caused. Acrylamide (AA) ${ }^{[22]}$ induced apoptosis in vivo and in vitro, accompanied by oxidative stress. Oxidative stress can induce autophagy in neurons in the brain. But if the ROS level in the cell exceeds the clearance range of autophagy, it will cause excessive autophagy and eventually lead to autophagic death. The study found that ${ }^{[23]} \mathrm{H}_{2} \mathrm{O}_{2}$ acting on hippocampal neurons HT22 cells, autophagy bodies gradually increased and eventually died with the long action of free radicals, suggesting that oxidative stress can induce hippocampal neuronal death. The hippocampus is a related brain region of learning, memory and emotion in the brain. Studies have confirmed that local changes in the hippocampus, such as volume reduction, neuronal apoptosis or death, are important causes of cognitive function changes.

\subsection{Tau proteins affect mitochondrial dys- function}

As a "power plant" in vivo, mitochondria play an important role in the formation of ATP through the process of oxidative phosphorylation. The damaged mitochondria can stimulate the production of a large number of reactive oxygen free radicals, which can cause oxidative damage to the central nervous system. The results showed that the expression of human tau protein could cause the dynamic imbalance of mitochondrial division and fusion between HEK293 cells and primary neurons, and the neuronal process was significantly shortened and the cell viability decreased ${ }^{[24]}$. Damage to mitochondrial function can affect mitochondrial endoplasmic reticulum (MAMs) structural coupling. Mitochondrial-endoplasmic reticulum structural coupling (MAMs) is a physical and biochemical connection between mitochondria and endoplasmic reticulum, and it is also a bridge between their structure and function. Studies have confirmed that changes in MAMs structure and function may be a key link in the pathogenesis of Parkinson's disease, Alzheimer's disease and other types of cognitive disorders, so MAMs injury is associated with cognitive dysfunction.

\subsection{Tau proteins affect endoplasmic reticu- lum-related degradation pathways}


The endoplasmic reticulum related degradation (ERAD) pathway refers to the process of degradation by the ubiquitin proteasome system (UPS) when the newly synthesized proteins or polypeptide molecules of the endoplasmic reticulum are non-folded proteins or misfolded proteins increase or cannot return to normal conformation. Abnormal tau protein accumulation can destroy endoplasmic reticulum related degradation pathway $^{[25]}$, hinder ubiquitin dependent proteasome degradation pathway, and then cause folding protein response. At the same time, endoplasmic reticulum stress is activated to induce neuronal apoptosis, which further affects cognitive function.

\subsection{Effects of tau proteins on oxidative stress}

Oxidative stress is a physiological process triggered by the breaking of the balance between reactive oxygen species and antioxidants, which can lead to lipid peroxidation, oxidative damage to proteins and DNA, and ultimately neuronal damage. Studies have shown that abnormal phosphorylation of tau proteins occurs $\mathrm{AD}$ the brain of patients and accumulates to form spiral filaments, that is, neuronal fiber tangles. However, hyperphosphorylated tau proteins lose the ability to bind to neuronal microtubules, resulting in the inability to regulate the structure and function of microtubules, thus causing neuronal axons to degenerate. The formation and growth of axons is the primary morphological change in neuronal differentiation, which makes cognitive functions such as information transmission, learning and memory more dependent on information transmission between neurons.

\section{Summary}

To sum up, tau protein is a microtubule-related protein of nerve cells, which has the function of synthesizing and stabilizing neurons and plays an important role in maintaining cell morphology, cell movement, signal transmission and other physiological processes. The NFT of hyperphosphorylation and aggregation of tau protein is one of the main pathological features in the brain of AD patients. Tau protein hyperphosphorylation is considered to be AD key pathogenic factor. The study found that the phosphorylation level of tau protein was increased in postoperative cognitive impairment, stroke with vascular cognitive impairment, diabetic encephalo- pathy and other cognitive dysfunction diseases, so a large number of studies believe that tau protein is involved in the regulation of cognitive impairment mechanism. The study found that the level of tau protein phosphorylation also increased during morphine dependence, so consider whether tau protein phosphorylation is involved in cognitive dysfunction caused by morphine dependence. This article reviews the overview of tau protein and its phosphorylation, its relationship with other cognitive disorders, tau protein and morphine dependence, and its possible effects on cognitive dysfunction caused by morphine dependence. In order to provide a new way of diagnosis and treatment for patients with cognitive dysfunction caused by morphine dependence and provide a new direction for the preparation of targeted drugs.

\section{References}

1. Zhang H, Wang H, Zhang M, et al. The influence of blood glucose fluctuation on hippocampal Tau protein phosphorylation of diabetic rats. Chinese Journal of Endocrinology and Metabolism 2017; 33(9): 776-780.

2. Puvenna V, Engeler M, Banjara M, et al. Is phosphorylated Tau unique to chronic traumatic encephalopathy? Phosphorylated Tau in epileptic brain and chronic traumatic encephalopathy. Brain Research 2016; 1630: 225-40.

3. Aribisala BS, Hernandez MCV, Royle NA, et al. Brain atrophy associations with white matter lesions in the ageing brain: The lothian birth cohort 1936. European Radiology 2013; 23(4): 1084-92.

4. Nikseresht S, Etebary S, Roodsari HRS, et al. The role of nitrergic system in antidepressant effects of acute administration of zinc, magnesium and thiamine on progesterone induced postpartum depression in mice. Tehran University Medical Journal 2010; 68(5): 261-267.

5. Miloi MM. The DsTau experiment: A study for Tau-neutrino production. Particles 2020; 3(1): 164 168.

6. Small GW, Siddarth P, Li Z, et al. Memory and brain amyloid and Tau effects of a bioavailable form of curcumin in non-demented adults: A double-blind, placebo-controlled 18-month trial. The American Journal of Geriatric Psychiatry 2018; 26(3): 266-277.

7. Ameri M, Shabaninejad Z, Movahedpour A, et al. Biosensors for detection of Tau protein as an Alzheimer's disease marker. International Journal of Biological Macromolecules 2020; 162: 1100-1108.

8. Horie K, Barthelemy NR, Mallipeddi N, et al. Regional correlation of biochemical measures of amyloid and tau phosphorylation in the brain. Acta Neuropathologica Communications 2020. 
9. Ma D. P1-099: CIG suppresses tau pathology in a mouse model of tauopathy through regulating the activity of PP2A. The Journal of the Alzheimer's Association 2019; 15(7S): 272-273.

10. Cao M, Liu F, Ji F, et al. Effect of c-Jun N-terminal kinase $(\mathrm{JNK}) / \mathrm{p} 38$ mitogen-activated protein kinase (p38 MAPK) in morphine-induced tau protein hyperphosphorylation. Behavioural Brain Research 2013; 237: 249-255.

11. Wang X, Zhao Y. The effect of AnshenDingzhi Fang on tau protein phosphorylation and BDNF/TrkB signaling pathway in Alzheimer's disease rats. Journal of Hainan Medical University 2019; 25(21): 1612-1616.

12. Frederiksen KS, Nielsen TR, Appollonio I, et al. Biomarker counselling, disclosure of diagnosis and follow-up in patients with mild cognitive impairment: A European Alzheimer' disease consortium survey. International Journal of Geriatric Psychiatry 2021; 36(2): 324-333.

13. Nicolia V, Ciraci V, Cavallaro RA, et al. GSK3 Fer-flanking DNA methylation and expression in Alzheimer's disease patients. Current Alzheimer Research 2017; 14(7): 753-759.

14. Huin V, Deramecourt V, Caparros-Lefebvre D, et al. The MAPT gene is differentially methylated in the progressive supranuclear palsy brain. Movement Disorders 2016; 31(12): 1883-1890.

15. Armentero MT, Sinforiani E, Ghezzi C, et al. Peripheral expression of key regulatory kinases in Alzheimer's disease and Parkinson's disease. Neurobiology of Aging 2011; 32(12): 2142-51.

16. Winston CN, Goetzl EJ, Schwartz JB, et al. Complement protein levels in plasma astrocyte-derived exosomes are abnormal in conversion from mild cognitive impairment to Alzheimer's disease dementia. Alzheimer's \& Dementia: Diagnosis, Assessment \& Disease Monitoring 2019; 11(1): 6166.

17. Chen X, Jiang H. Tau as a potential therapeutic tar- get for ischemic stroke. Aging 2019; 11(24): 12827-12843.

18. Tang SC, Yang $\mathrm{KC}$, Chen $\mathrm{CH}$, et al. Plasma h-Yueh,Chiu Ming-Japroteins in patients with vascular cognitive impairment. Neuromolecular Medicine 2018; 20(4): 498-503.

19. Viisanen H, Lilius TO, Sagalajev B, et al. Neurophysiological response properties of medullary pain-control neurons following chronic treatment with morphine or oxycodone: Modulation by acute ketamine. Journal of Neurophysiology 2020; 124(3): 790-801.

20. Yang S. Study on the role and mechanism of Tau and MAP-2 in CCK-8 mitigation of morphine-induced spatial memory damage (in Chinese) [PhD thesis]. Shijiazhuang: Hebei Medical University; 2013.

21. Ding K. Effect of the change of ghrelin and its receptor on accelerating diabetic thy by blood glucose fluctuation in GK rats. China Medical Abstracts (Internal Medicine) 2017; (1).

22. Xiao X, Sun J, Li S, et al. Acrylamide induced apoptosis in VSC4.1 cells through endoplasmic reticulum stress. Journal of Environmental \& Occupational Medicine 2017; 34(12): 1087-1092.

23. Cao X, Qi X, Wang S. Protective effects of astaxanthin on hippocampal neurons damage induced by hydrogen peroxide. Chinese Journal of Marine Drugs 2012; 31(6): 45-49.

24. Szabo L, Eckert A, Grimm A. Insights into disease-associated tau impact on mitochondria. International Journal of Molecular Sciences 2020; 21(17): 6344.

25. Abisambra J, Jinwal U, Blair L, et al. O2lues, Mathew Cockman, Amirthaa Suntharalingham, Pengfei Li, Ying Jin, Christopher Atkins, Chad Dickeymemo-associated degradation. The Journal of the Alzheimer's Association 2013; 9(4S): 329330. 